\title{
Local food-based complementary feeding recommendations developed by the linear programming approach to improve the intake of problem nutrients among 12-23-month-old Myanmar children
}

\author{
Lwin Mar Hlaing ${ }^{1,2 *}$, Umi Fahmida ${ }^{2}$, Min Kyaw Htet $^{3}$, Budi Utomo ${ }^{4}$, Agus Firmansyah ${ }^{5}$ and \\ Elaine L. Ferguson ${ }^{6}$ \\ ${ }^{1}$ Department of Public Health, National Nutrition Center, Ministry of Health, Nay Pyi Taw 100604, Myanmar \\ ${ }^{2}$ South East Asian Ministers of Education Organization Regional Center for Food and Nutrition (SEAMEO RECFON), \\ University of Indonesia, Jakarta 10430, Indonesia \\ ${ }^{3}$ The Center of Community Health and Population Studies, The Research Institute, Trisakti University, Jakarta 11440, Indonesia \\ ${ }^{4}$ Department of Population and Biostatistics, Faculty of Public Health, University of Indonesia, Jakarta 10430, Indonesia \\ ${ }^{5}$ Department of Child Health, Gasterohepatology Division, Faculty of Medicine, University of Indonesia, Jakarta 10430, Indonesia \\ ${ }^{6}$ London School of Hygiene and Tropical Medicine, The Faculty of Epidemiology and Population Health, London WC1E 7HT, UK
}

(Submitted 21 September 2014 - Final revision received 25 October 2015 - Accepted 2 November 2015 - First published online 23 December 2015)

\begin{abstract}
Poor feeding practices result in inadequate nutrient intakes in young children in developing countries. To improve practices, local food-based complementary feeding recommendations (CFR) are needed. This cross-sectional survey aimed to describe current food consumption patterns of 12-23-month-old Myanmar children ( $n$ 106) from Ayeyarwady region in order to identify nutrient requirements that are difficult to achieve using local foods and to formulate affordable and realistic CFR to improve dietary adequacy. Weekly food consumption patterns were assessed using a 12-h weighed dietary record, single 24-h recall and a 5-d food record. Food costs were estimated by market surveys. CFR were formulated by linear programming analysis using WHO Optifood software and evaluated among mothers ( $n 20)$ using trial of improved practices (TIP). Findings showed that $\mathrm{Ca}, \mathrm{Zn}$, niacin, folate and Fe were 'problem nutrients': nutrients that did not achieve $100 \%$ recommended nutrient intake even when the diet was optimised. Chicken liver, anchovy and roselle leaves were locally available nutrientdense foods that would fill these nutrient gaps. The final set of six CFR would ensure dietary adequacy for five of twelve nutrients at a minimal cost of $271 \mathrm{kyats} / \mathrm{d}$ (based on the exchange rate of $900 \mathrm{kyats} / \mathrm{USD}$ at the time of data collection: 3rd quarter of 2012), but inadequacies remained for niacin, folate, thiamin, Fe, $\mathrm{Zn}$, Ca and vitamin $\mathrm{B}_{6}$. TIP showed that mothers believed liver and vegetables would cause worms and diarrhoea, but these beliefs could be overcome to successfully promote liver consumption. Therefore, an acceptable set of CFR were developed to improve the dietary practices of 12-23-month-old Myanmar children using locally available foods. Alternative interventions such as fortification, however, are still needed to ensure dietary adequacy of all nutrients.
\end{abstract}

Key words: Complementary feeding recommendations: Linear programming: Optifood: Problem nutrients: Myanmar children

The first $1000 \mathrm{~d}$ of life, from conception until the child's second birthday, are considered the critical window of opportunity for preventing undernutrition and its long-term consequences. Poor complementary feeding practices during this critical window (between 6 and 24 months of age) increase the risk of undernutrition, morbidity and mortality in infants and young children $^{(1)}$. In Southeast Asian (SEA) countries, complementary feeding diets are rice-based and contain negligible amounts of animal source foods partly because of inaccessibility to or lack of knowledge about nutrient-rich foods as a result of economic constraints $^{(2)}$. These plant-based diets have a high content of anti-nutrients, such as phytate, that compromise the bioavailability of $\mathrm{Fe}$ and $\mathrm{Zn}^{(3)}$. In addition, impoverished populations often feed watery porridges to children under 2 years of age, which restricts the amount of food they can consume because of their limited gastric capacity, and increases their risk for inadequate intakes of energy and nutrients ${ }^{(4-6)}$. The plant-based nature of complementary foods used in developing countries is

Disclaimer: Publication of these papers was supported by unrestricted educational grants from PT Sarihusada Generasi Mahardhika and PT Nutricia Indonesia Sejahtera. The papers included in this supplement were invited by the Guest Editors and have undergone the standard journal formal review process. They may be cited. The Guest Editors declare that there are no conflicts of interest.

Abbreviations: CFR, complementary feeding recommendations; FBR, food-based recommendations; MFE, meat, fish, poultry and eggs; RNI, recommended nutrient intakes; TIP, trial of improved practices; WDR, weighed dietary record.

* Corresponding author: L. M. Hlaing, fax +9567431258, +9567431259, email lmhlaing78@gmail.com 
frequently associated with dietary micronutrient deficits, especially of $\mathrm{Fe}, \mathrm{Zn}$ and $\mathrm{Ca}^{(7-9)}$. It is therefore not surprising that multiple biochemical indicators of micronutrient deficiencies are frequently reported among infants and toddlers from developing countries ${ }^{(10,11)}$.

To tackle micronutrient deficiencies, either of or a combination of fortification, supplementation and dietary diversification is often used as the main strategy. Micronutrient fortification in staple foods or condiments, such as rice fortification, oil fortification or salt iodisation, is being implemented in countries to prevent micronutrient deficiencies in the general population. In contrast, fortification of specific foods, such as complementary food products, is being practised for specific target groups. Supplementation with single or multiple micronutrients is also effectively used to treat micronutrient deficiencies. Communication on behavioural change to promote dietary diversification is one of the strategies for improving the nutrient intake to prevent micronutrient deficiencies. At present, there is growing interest to promote the use of locally available nutrient-dense foods and improve complementary feeding practices, as it may be more sustainable and can tackle the co-existence of multiple micronutrient deficiencies ${ }^{(10,12,13)}$. However, questions remain about whether a food-based approach alone will ensure dietary adequacy and whether an effective behavioral change strategy is required for intervention success.

An approach based on linear programming (LP) analysis was recently developed to formulate population-specific complementary feeding recommendations (CFR) and identify nutrients for which dietary adequacy cannot be ensured using a realistic food-based approach (i.e. problem nutrients) ${ }^{(14)}$. In this approach, multiple factors are taken into account, including existing dietary patterns, food costs and cultural factors, to ensure the development of affordable, culturally acceptable $\mathrm{CFR}^{(14)}$. The LP approach has been used to assess whether combinations of local foods, to be used as home-prepared diets for children with severe malnutrition, can achieve the nutrient density levels of F100 in Ghana, Bangladesh and Latin America ${ }^{(15)}$. The findings showed that optimal combinations of local foods are unlikely to achieve the nutrient density level of F100 for vitamin E, riboflavin, thiamin, niacin, Zn, $\mathrm{Ca}$ and $\mathrm{Cu}$. LP was used in Indonesian and Cambodian studies to develop CFR and to identify nutrients that will likely remain low in their diets, and to test whether special formulated complementary food products will ensure dietary adequacy for young children $^{(7,16,17)}$. The findings revealed that combinations of locally available foods could improve the nutrient adequacy of their complementary feeding diets but could not entirely fill the nutrient gaps $^{(16)}$. However, to our knowledge, the acceptability of CFR developed using the LP approach has not yet been reported.

In Myanmar, the 2010 nation-wide survey showed that only $56.5 \%$ of infants received the recommended frequency of complementary feeding for their age (Multiple Indicator Cluster Survey (MICS) indicator 18, frequency of complementary feeding) and only $41 \%$ infants were adequately fed (MICS indicator 19 , adequately fed infants) ${ }^{(18)}$. Their complementary feeding diets were primarily based on grains and legumes, which would likely have a high phytate content that would compromise $\mathrm{Fe}$ and $\mathrm{Zn}$ bioavailability ${ }^{(19)}$. These suboptimal feeding practices are associated with a high risk for micronutrient deficiencies.
Affordable, culturally appropriate CFR have never been developed for young children in Myanmar. It is also not known whether CFR based on nutrient-dense locally available foods will fill the nutrient gaps in the diets of these children and whether they can be successfully adopted. In this study, therefore, we developed a set of CFR based on locally available foods using LP, and undertook formative research, using 'trial of improved practices' (TIP), to elucidate the barriers and motivating factors for behavioural change ${ }^{(7)}$. The specific objectives of this study were to develop population-specific local food-based CFR for 12-23-month-old Myanmar children who are at high risk for undernutrition, to identify problem nutrients in their diet and to investigate the acceptability and feasibility of the final CFR.

\section{Methods}

Study site

This study was conducted in Pantanaw and Nyaungdon townships of the Ayeyarwady Region, which are located 95.1 and $66 \mathrm{~km}$, respectively, to the north-west of the old capital city (Yangon) of Myanmar. These two townships were purposively selected because of the high prevalence of both acute and chronic undernutrition among preschool children (unpublished results). The study was undertaken in two randomly selected villages (Inn-ma village and Sarmalauk village) from Pantanaw and Nyaungdone townships.

\section{Study design}

A cross-sectional survey was conducted from June to September 2012 to assess the food consumption patterns and anthropometric status of 12-23-month-old children. A market survey was carried out to determine the cost per $100 \mathrm{~g}$ of edible portion of local foods commonly consumed by 12-23-month-old children. The feasibility of adopting the CFR developed from the crosssectional dietary data was evaluated using TIP. This study was the formative phase of a randomised, placebo-controlled, community-based intervention trial (ClinicalTrials.gov Identifier: NCT01758159) designed to evaluate the effectiveness of CFR promoted with or without Fe supplementation in order to improve the micronutrient status, growth and gut microbiota composition of Myanmar children.

\section{Ethical approval}

Ethical approval was obtained from The Ethical Committee on Medical Research involving Human subjects, Department of Health, Ministry of Health, Myanmar' (Letter No: Ethical Committee 1/2012-505), and verbal informed consent was obtained from all respondents who participated in the study.

\section{Study participants}

All mothers living in Inn-ma and Sarmalauk villages who had a 12-23-month-old child were invited to participate in the study. To be included in the study the child had to be between 12 and 
23 months of age inclusive, apparently healthy and not suffering from any illness that affected their dietary intakes. After excluding two children who did not meet the inclusion criteria and ten children whose mothers did not agree to participate in the study, 108 mother-child pairs were recruited to participate in the current survey. Food consumption data were obtained only from 106 children after excluding two more children who had severe wasting.

\section{Data collection}

Socio-demographic characteristics of the subjects. The age of the children was obtained from the birth registration card or the immunisation card. Using an interviewer-administered questionnaire, information on socio-demographic characteristics, breast-feeding and complementary feeding practices was collected through face-to-face interviews with the mothers.

Anthropometry. Duplicate measurements of each child's weight and recumbent length were recorded using standard anthropometric techniques ${ }^{(20)}$. The body weight of the children was measured using the Salter scale (Salter spring dial scale Model 2356 S , precision $\pm 1 \mathrm{~g}$; Salter) with the child minimally clothed. Recumbent length was measured using a wooden length board (Wooden length board, precision $\pm 0 \cdot 1 \mathrm{~cm}$; UNICEF Supply) ${ }^{(20)}$. Six enumerators were trained to take anthropometric measurements and they were standardised by the principal investigator (L. M. H.). The four enumerators who had the highest measurement precision and accuracy and met the required standard for length and weight measurements were selected as the primary anthropometrists ${ }^{(21)}$, whereas the others assisted in holding the children.

Anthropometric and socio-demographic data analysis. Anthropometric data were analysed using the WHO Anthro Software (version 2.0.4) to generate the children's $Z$-scores for length-for-age (LAZ), weight-for-length (WLZ) and weight-for-age (WAZ). The children were classified as stunted, wasted or underweight if their LAZ, WLZ and WAZ were less than -2 SD and as severely stunted, wasted or underweight if the respective $Z$-scores were less than $-3 \mathrm{sD}$ according to the World Health Organization $^{(22)} 2006$ multicentre growth reference standards. The percentage of children fed according to the World Health Organization ${ }^{(23)}$-recommended infant and young child feeding practices was calculated, which included early initiation of breastfeeding, colostrum feeding, currently breast-feeding, exclusive breast-feeding upto 6 months of age, pre-lacteal feeding, and the age of introduction of solid, semi-solid or soft foods in addition to breast milk. All means and standard deviations and percentages for anthropometric and socio-demographic data were analysed by SPSS for Windows (version 16.0; SPSS).

Food consumption pattern. The dietary intakes of the children were assessed using a 12-h weighed dietary record (WDR), 12-h (with serving size estimates) and 24-h (without serving size estimates) dietary recalls and a 5-d food record as described in a previous study ${ }^{(7)}$. Food consumption data from the WDR were used to estimate the average serving sizes of foods consumed by these children, and data from all dietary assessment methods were used to describe the median and range of food consumption patterns in $7 \mathrm{~d}$.

In the WDR, all foods and beverages consumed by the child between 06.00 and 18.00 hours (or until the last meal on the day of weighing) were weighed (Tanita digital scale for kitchen use, model KD-160, precision $\pm 1 \mathrm{~g}$; Tanita Corporation) and recorded by five trained enumerators. A 12-h recall for all foods and beverages consumed from 18.00 hours on that day to 06.00 hours the next morning was also collected. Recalled food serving sizes were estimated by asking the mother to estimate the amount in local cups or utensils, and the estimated amounts were weighed using the real food models. The WDR and 12-h recall were proportionately collected on all days of the week to account for the effect of any day of the week on dietary intakes at the population level.

A single 24-h recall was performed to obtain information on the frequency of foods and beverages consumed within the $24 \mathrm{~h}$ before the WDR day. Mothers were interviewed to get the list of all foods and beverages consumed by the child the previous day. Information was also collected on the time of consumption, but the place of consumption and the amount of foods/beverages consumed were not estimated. The frequency of foods and beverages consumed after the WDR day was also assessed using a self-administered 5-d food tally. In this method, mothers were asked to record all foods and beverages consumed by her child during the 5 -d period. Food tallies were collected every day and checked by two trained local volunteers in each village. For illiterate mothers and those who forgot to record the foods consumed, the volunteers conducted a 24-h recall using methods identical to those described above.

In-depth interviews were also conducted with all recruited mothers by written-recording and with six local women leaders by tape-recording to understand the food taboos and cultural beliefs related to the complementary feeding practices, as well as seasonal variation in complementary feeding practices.

Market surveys. A market survey was conducted in one local market, which the mothers commonly used, in each village. In this survey, two enumerators purchased three samples per market of all foods consumed by the children. For snacks and ready-to-eat foods, the cost per $100 \mathrm{~g}$ of edible portion was directly calculated. For cooked foods or composite dishes, each raw ingredient was weighed and their costs were summed to obtain the cost for all ingredients. The final cooked food was also weighed, and the cost per $100 \mathrm{~g}$ of cooked food or composite dish was then calculated. These data were used to define model parameters in the LP analyses.

Food composition database. The Myanmar food composition database is not complete for all nutrients analysed in this study. Any missing energy or nutrient contents were imputed mainly from the Indonesian food composition database, www. nutrisurvey.de, but also from Malaysian, Singaporean and United States Department of Agriculture food composition databases by matching the foods using key nutrients as described by Bunch \& Murphy ${ }^{(24)}$. For the composite dishes and those that 
are unique to Myanmar, recipe data were collected by asking ten mothers to cook these local dishes. Each raw ingredient, including water and condiments, was weighed individually and recorded. The final cooked food was also weighed and recorded. The nutrient contents of cooked food per $100 \mathrm{~g}$ was calculated on the basis of the nutrient content of the total cooked food multiplied by 100 and divided by the total weight of the cooked food. The same procedure was carried out for all ten mothers for each dish, and the average value was taken.

Preparation of linear programming model parameters. The dietary survey data were used to define the LP model parameters. Preparation of LP model parameters was done in Microsoft Excel 2007. These parameters included a list of foods consumed by the children (from all dietary assessment methods), an average serving size for each food (from the WDR, median serving size in $\mathrm{g} / \mathrm{d}$ for consumers only) and the food patterns (from all dietary assessment methods) defined as number of servings per week for individual foods, food groups and food subgroups. The range in weekly food pattern consumption frequencies (minimum, average and maximum) was defined as the 10th, 50th and 90th percentile of the frequency distribution for each food item, food group and subgroup. For nutrient-dense foods for which the 90th percentile value was 0 (i.e. $<10 \%$ of children consumed it), the maximum frequency of consumption was defined as its 95th or 99th percentile with the aim to promote consumption of these nutrient-dense foods.

These LP parameters were used to set up the LP models for the analyses in the WHO Optifood software (version 3.1.1.0) ${ }^{(25)}$. Food items consumed by the children were categorised into the food groups and subgroups used in Optifood. In all, thirteen nutrients were analysed in Optifood: protein, fat, Ca, vitamin C, thiamin, riboflavin, niacin, vitamin $\mathrm{B}_{6}$, folate, vitamin $\mathrm{B}_{12}$, vitamin A, Fe and Zn. The energy constraint used in all LP models was equal to the average energy requirements of the children calculated from the WHO/FAO energy requirement algorithm using the mean body weight of $9.2 \mathrm{~kg}$.

Development of complementary feeding recommendations and identification of problem nutrients. Food-based CFR were developed and tested by using the WHO Optifood in four analytical modules as described by Daelmans et al. ${ }^{(25)}$. Model parameters were checked in Optifood's module I, and all analyses were carried out in Optifood's modules II and III. In the module II analysis, two nutritionally best diets were generated in which one conformed to the target population's average food patterns (i.e. the 'food pattern best diet'), whereas the other could deviate from the average food patterns while still remaining within the observed food pattern ranges (i.e. the "no food pattern best diet'). In the module III analysis, the diets that had the lowest (i.e. nutrients minimised to identify the worstcase scenarios) and the highest (i.e. nutrients maximised to identify the best-case scenarios) nutrient contents were first generated without CFR to provide baseline levels for comparison. In subsequent module III analyses, the worst-case scenario nutrient levels, for each alternative CFR tested, were generated and compared.
The problem nutrients and dietary adequacy were defined using the $\mathrm{FAO} / \mathrm{WHO}$ recommended nutrient intakes (RNI) for all nutrients ${ }^{(19)}$, except $\mathrm{Zn}$, for which the International Zinc Nutrition Consultative Group's RNI was used ${ }^{(26)}$. Moderate bioavailability for $\mathrm{Zn}$ (RNI for $\mathrm{Zn}=3 \mathrm{mg} / \mathrm{d}$ ) and $10 \%$ bioavailability for Fe (RNI for Fe $=5.8 \mathrm{mg}$ ) were assumed on the basis of the diet patterns of the study population, which included unrefined cereal-based diets with a small amount of animal source foods (i.e. commonly fish) ${ }^{(16,19,26)}$. The nutrients that did not achieve $100 \%$ RNI in both best diets (in module II) were defined as 'problem nutrients' and those that did not achieve $100 \%$ RNI in the 'best-case scenario' (maximised) diets (module III; without CFR) were defined as 'absolute problem nutrients $^{,(16)}$. When evaluating CFR, the dietary adequacy for each nutrient was defined as a 'worst-case scenario' (minimised) nutrient level $>65 \%$ of RNI (module III, minimised nutrient contents). ${ }^{(16)}$

Foods rich in problem nutrients were identified on the basis of the food patterns of the best diets generated in module II to formulate alternative sets of CFR in the module III analyses. Each set of CFR was expressed as the number of daily servings per week to be consumed of individual food groups, subgroups, food items or combinations of them. The set of CFR that achieved dietary adequacy for the high number of nutrients at the lowest possible cost (module III analyses comparisons) was selected as the final set of CFR. CFR messages, for these final CFR, were written in a simple way in the local language that could be easily understood by the mothers.

Trial of improved practice. Household trials were conducted with twenty mothers (ten mothers from each village) to investigate the acceptability and feasibility of the final CFR. TIP were conducted by means of two visits to each of the twenty households, with a 1-week interval. During the first visit, the CFR were explained to the mothers using picture charts as visual aids. Other health messages were also given, including the causes of diarrhoea and worm infestation and the importance of using boiled drinking water, personal hygiene and sanitation. The mothers/caretakers were then encouraged to try to practise the CFR for the next $7 \mathrm{~d}$. In the follow-up visit, an in-depth interview was carried out to explore the challenges the mothers faced when practising the CFR, their reaction to feeding the child according to CFR, and the response from the child and other household members on practising the CFR and the benefits to putting CFR into practice. In addition, child feeding was also observed in two mother-child pairs to ascertain the response of the child during feeding.

\section{Results}

Selected socio-demographic characteristics and anthropometric status of subjects

The medians income of the families was 90000 (60000$150000)$ kyats/month (approximately $90 \mathrm{USD} / \mathrm{month}$; approximately $60-150$ USD/month). Almost $70 \%$ of families used unboiled water from the river or tube well for drinking purpose. 
The mean age of the children was $19 \cdot 0$ (sD $4 \cdot 1$ ) months and $47 \%$ were boys. The mean body weight of the children was $9.2 \mathrm{~kg}$ and length was $77.3 \mathrm{~cm}$. Out of 108 children, stunting, wasting and underweight were found in $35.2 \%(n 38), 10 \cdot 2 \%(n 11)$ and $25.9 \%$ ( $n$ 28), and severe stunting, wasting and underweight were found in 10.2\% ( $n$ 11), 1.9\% ( $n$ 2) and 9.3\% ( $n$ 10), respectively. Children with severe undernutrition were referred to the local health staff for necessary treatment; two children with severe wasting were referred to the township hospital and were not included in the dietary survey.

\section{Breast-feeding and complementary feeding practices}

An overall $78.7 \%$ of the children were still being breast-fed at the time of the survey; breast-feeding was initiated early in $87 \%$ and almost all children had been fed colostrum. Only $12.9 \%$ received pre-lacteal feeding, which was mainly formula milk $(57 \cdot 1 \%)$ and rice gruel $(14 \cdot 3 \%)$. Nearly half of the children ( $45.4 \%$ ) had been exclusively breast-fed upto 6 months of age and the mean age of introduction of complementary food was 6.1 (sD 4.2) months (range 1-18 months).

\section{Food frequency, food serving sizes and food patterns}

Table 1 shows the food group and subgroup consumption patterns of the children. The median (50th percentile) food group pattern included 7 servings/week of grains and grain products as staple foods, 3 servings/week of animal proteins and bakery and breakfast cereals, and $<1$ serving/week of plant proteins, vegetables, fruits, dairy products, starchy roots and tubers. The children consumed 153 food items, of which six foods were staple foods (grains and grain products), thirty-eight were animal source foods (MFE: meat, fish, poultry and eggs), ten were plant protein sources (legumes, nuts and seeds), twenty-one were vegetables, three were roots and tubers, twenty-four were composites (e.g. coconut noodle (main ingredients are coconut milk, chickpeas, chicken and noodle), Mote-hin-gar (main ingredients are fish, chickpeas, rice flour and stem of banana plant and vermicelli), mixed vegetable and chickpea soup (common ingredients are chickpeas, okra, gourd and eggplant), fried noodle (main ingredients are noodles, chicken, egg and cabbage)), eleven were fruits, ten were bakery and breakfast cereals, seven were sweetened snacks and desserts, three were savoury snacks, five were dairy products, nine were beverages, three were added sugars, and another three were from the miscellaneous food group (Table 1).

None of the foods or beverages consumed by the children was recognised by the mothers as fortified products. However, we found that imported cereal-based beverages consumed, such as Milo and Ovaltine, seemed to be fortified products based on the nutrient facts on the packaging. These cerealbased beverages were consumed by $12 \%$ ( $n$ 13) of children, whereas the amount consumed was $20 \mathrm{~g}$ (one sachet) a day on average and the frequency of feeding varied widely from 1 to 6 times/week. Nutrient facts on the packaging mentioned that a $20 \mathrm{~g}$ sachet of these cereal-based beverages contained about $8 \% \mathrm{RNI}$ of folate, about $25 \% \mathrm{RNI}$ of $\mathrm{Fe}$, about $35 \% \mathrm{RNI}$ of $\mathrm{Zn}$, about $40 \%$ RNI of niacin, about $55 \%$ RNI of $\mathrm{Ca}$ and about $70 \%$ RNI of vitamin $\mathrm{B}_{6}$. Despite such nutrient labelling, laboratoryconfirmed tests for quality assurance of these nutrient contents are not yet available inside the country.

From the MFE group, the majority of children (94.3\%) were fed fish, which was readily available and affordable (or free) throughout the year. Eggs were consumed by more than half of the children ( $58.5 \%$ ), poultry (especially chicken) by $45.3 \%$, and sea foods were consumed by $46 \cdot 2 \%$. Although available in local markets, only $3.7 \%(n)$ and $14.2 \%(n 15)$ of mothers fed liver and red meat to their children, respectively. The main reason for not feeding red meat to the children was cost constraint: 400 kyats (approximately 0.33 USD)/100 $\mathrm{g}$ of chicken liver and red meat. For liver, it was a belief among the mothers that liver led to worm infestation. Although $44.3 \%$ consumed vegetables, the amount fed and frequency of feeding were quite small as vegetables were believed to cause diarrhoea in children. Fruits were consumed by $50.9 \%$ of the children, of

Table 1. Number of foods consumed and the food group consumption patterns of 12-23-month-old study participants (Number of servings per week; 10th, 50th and 90th percentiles)

\begin{tabular}{lcccc}
\hline & & \multicolumn{3}{c}{ Frequency/week } \\
\cline { 2 - 5 } Food groups & $\begin{array}{c}\text { No. of foods } \\
\text { consumed }\end{array}$ & $\begin{array}{c}\text { 10th percentile } \\
\text { (minimum) }\end{array}$ & $\begin{array}{c}\text { 50th percentile } \\
\text { (average) }\end{array}$ & $\begin{array}{c}\text { 90th percentile } \\
\text { (maximum) }\end{array}$ \\
\hline 1. Grains and grain products & 6 & 6 & 7 & 14 \\
2. Meat, fish and eggs & 38 & 0 & 3 & 7 \\
3. Vegetables & 21 & 0 & $0 \cdot 1$ & 4 \\
4. Legumes, nuts and seeds & 10 & 0 & $0 \cdot 1$ & 4 \\
5. Composites & 24 & 0 & $0 \cdot 1$ & 3 \\
6. Roots and tubers & 3 & 0 & $0 \cdot 1$ & 6 \\
7. Fruits & 11 & 0 & $0 \cdot 1$ & 6 \\
8. Bakery and breakfast cereals & 10 & 0 & 3 & 2 \\
9. Sweetened snacks and desserts & 7 & 0 & $0 \cdot 1$ & 1 \\
10. Savoury snack & 3 & 0 & $0 \cdot 1$ & 7 \\
11. Dairy products & 5 & 0 & 1 & 1 \\
12. Beverages & 9 & 0 & 0 & 1 \\
13. Added sugar & 3 & 0 & 0 & 2 \\
14. Miscellaneous & 3 & 0 & & 2 \\
\hline
\end{tabular}


Table 2. Serving sizes (consumers only) and upper constraint level (maximum serving per week) for modelled food items (Medians and ranges)

\begin{tabular}{|c|c|c|c|}
\hline \multirow[b]{2}{*}{ Nutrient-dense foods included in the model } & \multicolumn{2}{|c|}{ Serving size $(g / d)$} & \multirow{2}{*}{$\begin{array}{l}\text { Upper constraint } \\
\text { (serving/week) }\end{array}$} \\
\hline & Median & Range & \\
\hline \multicolumn{4}{|l|}{ Grains and grain products } \\
\hline Rice & 100 & $20-380$ & 7 \\
\hline \multicolumn{4}{|l|}{ Meat, fish and eggs } \\
\hline Red meat (beef) & 14 & $11-16$ & 1 \\
\hline Poultry (chicken) & 24 & $20-25$ & 2 \\
\hline Organ meat (chicken liver) & 30 & $20-40$ & $1^{*}$ \\
\hline Fish without bones (carp fish) & 31 & $11-82$ & 7 \\
\hline Small whole fish with bones (anchovy) & 10 & $18-37$ & 1 \\
\hline Eggs (chicken or duck eggs) & 27 & 13-38 & 4 \\
\hline \multicolumn{4}{|l|}{ Legumes, nuts and seeds } \\
\hline Cooked beans and lentils (chickpea soup) & 26 & $3-59$ & 2 \\
\hline Nuts and seeds (peanuts) & 16 & $10-28$ & 1 \\
\hline \multicolumn{4}{|l|}{ Vegetables } \\
\hline Roselle leaves (fried) & 40 & $35-50$ & $1^{*}$ \\
\hline \multicolumn{4}{|l|}{ Fortified cereal-based beverages } \\
\hline Ovaltine/Milo & 25 & $10-30$ & 6 \\
\hline
\end{tabular}

* 95 th Percentile for those foods for which the 90th percentile is still 0.

which $20 \cdot 8 \%$ were ripe mangoes, which was a seasonal fruit during the data collection period (rainy season). Legumes, nuts and seeds were consumed by $67.9 \%$ of the children and were mainly cooked or boiled beans even though there was a belief that this food group can cause childhood diarrhoea. Milk and dairy products were consumed by $29.2 \%$ of children.

The food serving sizes were highly varied between individual children or between different types of foods. Table 2 lists the serving sizes and frequency of consumption per week (upper constraint) for staple food (rice) and for some foods that are rich in 'problem nutrients' ( $\mathrm{Ca}, \mathrm{Zn}$, niacin, folate and $\mathrm{Fe}$ ). The median serving sizes of foods within food groups are 85 (range 22-244) g/d for grains and grain products, 26 (range 11-57) g/d for MFE, 37 (range 10-59) $\mathrm{g} / \mathrm{d}$ for legumes, nuts and seeds, 30 (range 8-40) g/d for fried vegetables, 50 (range 45-125) g/d for vegetable soups, 31 (range 10-71) g/d for fruits and 57 (range $15-117) \mathrm{g} / \mathrm{d}$ for dairy products. The children consumed 2-3 staples/d, and 1-5 snacks/d, which were mostly from the sweetened, savoury and miscellaneous snacks food groups and less from the fruits, roots and legumes food groups.

\section{Problem nutrients and food-based complementary feeding recommendations}

Among the two best diets formulated in module II, the 'food pattern best diet' cost 335 kyats/d (approximately $0.35 \mathrm{USD} / \mathrm{d}$ ), which recommended at least 12 servings/week of staples, 1 serving/d of MFE and 4 servings/week of bakery and breakfast cereals in addition to breast milk every day. 'No food pattern best diet' cost 423 kyats/d (approximately $0.44 \mathrm{USD} / \mathrm{d}$ ), which recommended 6 servings/week of bakery and breakfast cereals, 4 servings/week of vegetables, 6 servings/week of fruits, 2 servings/d of staples and 1 serving/d of MFE and breast milk. Ca, $\mathrm{Zn}$, niacin, folate and Fe were identified as 'problem nutrients' in the two best diets (i.e. nutrient level in the two best diets is $<100 \%$ RNI) (Table 3, best diets: first two rows), and all 'problem nutrients' except Fe were 'absolute problem nutrients' (i.e. nutrient level in the best-case scenario diet without a CFR is $<100 \% \mathrm{RNI}$ ) (Table 3, best-case scenario without a CFR).

Potential nutrient-dense food sources identified that could be promoted to help achieve dietary adequacy were chicken liver, anchovies (small fish with edible bones) and roselle leaves. Other good sources of nutrients in module II were the nutrientdense food groups and subgroups of fortified cereal-based beverages (i.e. Ovaltine, Milo and other cereal drinks), cooked beans and lentils, and eggs. These food items, groups and subgroups were incorporated either separately or in combination into the first draft CFR to produce alternative sets of CFR to test in module III.

In all, twelve alternative sets of CFR were produced and compared on the basis of their worst-case scenario nutrient levels and diet cost in module III. These alternative CFR did not meet $65 \%$ of RNI for all nutrients except protein, vitamin $\mathrm{C}$, vitamin $\mathrm{A}$, riboflavin and vitamin $\mathrm{B}_{12}$. Of these alternative CFR, CFR 2, 3 and 4 were the most promising because they achieved $>40 \% \mathrm{RNI}$ for $\mathrm{Fe}, \mathrm{Zn}$ and $\mathrm{Ca}$, about $30 \% \mathrm{RNI}$ for folate and $>25 \%$ RNI for niacin (Table 3; alternative sets of CFR, worstcase scenarios). Of these three CFR, CFR 4 was selected as the final CFR as its worst-case scenario nutrient levels achieved the highest levels for all absolute problem nutrients at a cost of 271 kyats/d (approximately 0.29 USD/d), which is cheaper than either CFR 2 or 3 (Table 3; alternative sets of CFR, worst-case scenarios). Table 4 shows the final CFR messages and the recommended minimum portion sizes for each CFR.

\section{Trial of improved practices}

The findings of TIP are presented in Table 5. During the first visit, the mothers were explained about the CFR and encouraged to feed their children according to CFR. The mothers were reluctant to feed their children with liver and roselle as they believed that liver can cause worm infestations and roselle can cause diarrhoea and abdominal discomfort in children. They were explained that worm infestation and diarrhoea are caused 
Table 3. Comparisons of nutrient levels and minimised costs of the two best diets (module II), worst-case and best-case scenario diets without complementary feeding recommendations (CFR) (module III), and alternative sets of CFR tested (module III; worst-case scenarios only)

\begin{tabular}{|c|c|c|c|c|c|c|c|c|c|c|c|c|c|}
\hline \multirow[b]{2}{*}{ Analysis } & \multicolumn{12}{|c|}{ Achievement of nutrients (\% RNI) } & \multirow[b]{2}{*}{ Cost (kyats/d) } \\
\hline & Protein & $\mathrm{Fe}$ & $\mathrm{Zn}$ & $\mathrm{Ca}$ & Niacin & Folate & Vit C & Thiamin & Riboflavin & Vit $\mathrm{B}_{6}$ & Vit $B_{12}$ & Vit $A$ & \\
\hline Best diet (food pattern) & $182 \cdot 2$ & $50 \cdot 3$ & $57 \cdot 7$ & 33.9 & $42 \cdot 0$ & $35 \cdot 6$ & $113 \cdot 0$ & $92 \cdot 2$ & $68 \cdot 2$ & $73 \cdot 2$ & $423 \cdot 8$ & $131 \cdot 3$ & 335 \\
\hline Best diet (no-food pattern) & $227 \cdot 6$ & $96 \cdot 3$ & $72 \cdot 0$ & $66 \cdot 4$ & $62 \cdot 1$ & $86 \cdot 7$ & $169 \cdot 3$ & $113 \cdot 3$ & $108 \cdot 7$ & $100 \cdot 0$ & $469 \cdot 7$ & $163 \cdot 8$ & 420 \\
\hline Best-case scenario without CFR & $266 \cdot 8$ & $102 \cdot 2$ & $78 \cdot 2$ & 72.4 & $79 \cdot 1$ & $90 \cdot 1$ & $241 \cdot 3$ & 139.4 & $139 \cdot 7$ & 137.5 & $488 \cdot 8$ & $188 \cdot 8$ & 665 \\
\hline Worst-case scenario without CFR & $111 \cdot 2$ & $15 \cdot 3$ & $31 \cdot 1$ & 28.5 & $6 \cdot 8$ & $1 \cdot 2$ & 62.4 & $20 \cdot 1$ & $16 \cdot 7$ & $39 \cdot 1$ & $54 \cdot 3$ & 0.0 & 216 \\
\hline \multicolumn{14}{|l|}{$\begin{array}{l}\text { Alternative sets of CFR tested } \\
\text { (worst-case scenarios only) }\end{array}$} \\
\hline CFR $1^{*}$ & $147 \cdot 6$ & 381 & $39 \cdot 6$ & $45 \cdot 9$ & $19 \cdot 0$ & $12 \cdot 7$ & $95 \cdot 4$ & $31 \cdot 7$ & $53 \cdot 9$ & $46 \cdot 6$ & $92 \cdot 1$ & $9 \cdot 6$ & 275 \\
\hline CFR $2 \dagger$ & $151 \cdot 8$ & 43.5 & $41 \cdot 6$ & $45 \cdot 9$ & $26 \cdot 3$ & $29 \cdot 6$ & $98 \cdot 7$ & $34 \cdot 1$ & $76 \cdot 1$ & $48 \cdot 1$ & $354 \cdot 0$ & $131 \cdot 0$ & 278 \\
\hline CFR $3 \ddagger$ & $152 \cdot 1$ & $43 \cdot 4$ & $41 \cdot 6$ & $45 \cdot 8$ & $26 \cdot 0$ & $29 \cdot 4$ & $95 \cdot 4$ & $33 \cdot 8$ & $76 \cdot 0$ & $47 \cdot 9$ & 354.5 & $130 \cdot 9$ & 275 \\
\hline CFR 4§ & $146 \cdot 6$ & 43.4 & 41.4 & $45 \cdot 8$ & $25 \cdot 9$ & 29.4 & 95.4 & $33 \cdot 8$ & $76 \cdot 0$ & $47 \cdot 8$ & 352.9 & $130 \cdot 9$ & 271 \\
\hline CFR $5 \|$ & 143.9 & $43 \cdot 2$ & $40 \cdot 1$ & $41 \cdot 0$ & $24 \cdot 0$ & $29 \cdot 1$ & $95 \cdot 7$ & 33.8 & 75.4 & $47 \cdot 4$ & 334.9 & 131.0 & 275 \\
\hline CFR 6ף & 138.8 & $43 \cdot 1$ & $40 \cdot 0$ & 41.0 & 24.0 & $29 \cdot 1$ & 95.4 & 33.8 & $75 \cdot 3$ & $47 \cdot 2$ & 333.9 & $130 \cdot 9$ & 269 \\
\hline
\end{tabular}

RNI, recommended nutrient intake; vit, vitamin.

* CFR 1 = Vegetables, 4 servings/week; fruits, 4 servings/week; fortified cereal-based beverages, 5 servings/week; cooked beans, 3 servings/week; eggs, 2 servings/week; fish without bones, 2 servings/week; anchovies, 1 serving/week; organ meat, 1 serving/week; roselle leaves, 1 serving/week.

† CFR 2 =Vegetables, 4 servings/week; fruits, 4 servings/week; fortified cereal-based beverages, 5 servings/week; cooked beans, 3 servings/week; eggs, 2 servings/week; fish without bones, 2 servings/week; anchovies, 1 serving/week; liver, 1 serving/week; roselle leaves, 1 serving/week.

‡ CFR 3 = Meat, fish and eggs, 7 servings/week; fortified cereal-based beverages, 5 servings/week; cooked beans, 3 servings/week; eggs, 2 servings/week; anchovies, 1 serving/ week; liver, 1 serving/week; roselle leaves, 1 serving/week.

§ CFR 4 =Fortified cereal-based beverages, 5 servings/week; cooked beans, 3 servings/week; eggs, 2 servings/week; anchovies, 1 serving/week; liver, 1 serving/week; roselle leaves, 1 serving/week.

|| CFR 5 = Vegetables, 4 servings/week; fortified cereal-based beverages, 5 servings/week; cooked beans, 3 servings/week; eggs, 2 servings/week; fish without bones, 2 servings/ week; liver, 1 serving/week; roselle leaves, 1 serving/week.

I CFR 6 = Fortified cereal-based beverages, 5 servings/week; cooked beans, 3 servings/week; eggs, 2 servings/week; liver, 1 serving/week; roselle leaves, 1 serving/week.

Table 4. Recommended frequency and portion sizes of the final complementary feeding recommendations formulated for 12-23-month-old Myanmar children from Ayeyarwady region

\section{Breast-feed daily on demand}

2. At least 3 main meals in a day

3. At least 1 serving/d of animal protein, including

a. At least 1 serving/week of liver

b. At least 1 serving/week of anchovy

c. At least 2 servings/week of eggs

4. Feed plant protein at least every alternate day, including

a. At least 3 servings/week of cooked beans and lentils

5. At least 1 serving/d of vegetables and or fruits, including

a. At least 1 serving/week of roselle leaves

6. At least 2 servings/d of snacks in between main meals, including

a. At least 5 servings/week of fortified cereal-based beverages (e.g. Milo, Ovaltine)

Recommended minimum portion size/ meal

1 serving of chicken liver $=30 \mathrm{~g}$ (cooked weight)

1 serving of anchovy $=10 \mathrm{~g}$ (fried weight)

1 serving of roselle $=40 \mathrm{~g}$ (cooked weight)

1 serving of fortified cereal-based beverages $=25 \mathrm{~g}$ (dry powder weight)

1 serving of cooked beans $=30 \mathrm{~g}$ (cooked weight)

1 serving of eggs $=25 \mathrm{~g}$ (boiled weight)

1 serving of rice $=100 \mathrm{~g}$ (cooked weight)

1 serving of fish without bones $=20 \mathrm{~g}$ (cooked weight)

1 serving of vegetables $=30 \mathrm{~g}$ (cooked weight)

1 serving of fruits $=30 \mathrm{~g}$ (wet weight)

by poor personal hygiene and unsafe drinking water. The mothers were also encouraged to wash their hands properly with soap and water and to use boiled water for drinking. During the follow-up visit, the challenges the mothers faced when practising the CFR during this 1-week trial were explored. The main challenge, which was reported by $55 \%(n 11)$ of mothers, was that the child could not finish the recommended portion sizes for vegetables as they did not want to chew the vegetables. Observation of child feeding also showed that the child frequently spit out the vegetables as he or she did not want to chew and could not swallow the vegetables. Therefore, we suggested that mothers prepare the vegetables in such a way that they were easy for the child to chew, such as chopping them into small pieces. Most mothers (75\%) complained that they needed more time to prepare foods separately for the child instead of following their usual practice of sharing the family food with the child. We informed the mothers that the family food could continue to be shared with the child and the importance of including a variety of food groups in their daily diet not only for the child but also for the whole family. The mothers felt that the cost of the diet for the child become more expensive when they practised the CFRs as compared with usual cost. We found out the reason was the additional cost of less-nutritious snacks such as crisps and soft drinks in addition to the cost of the CFR diet in which the cost of snacks was already included. The children were commonly fed these snacks and we advised the mothers not to feed the children such less-nutritious snacks, but instead to feed the child nutritious snacks such as fruits, roots and legumes. Four mothers (20\%) did not feed their child liver: two because the grandmothers did not allow it as a result of their strong belief that liver caused worm infestations; and two because mothers could not afford to buy liver or other animal source foods other than fish that they caught themselves. Despite these challenges, several motivating factors to continue practising the CFR were also identified. All mothers said they believed that their child would have a balanced and nutritious diet if they were fed according 


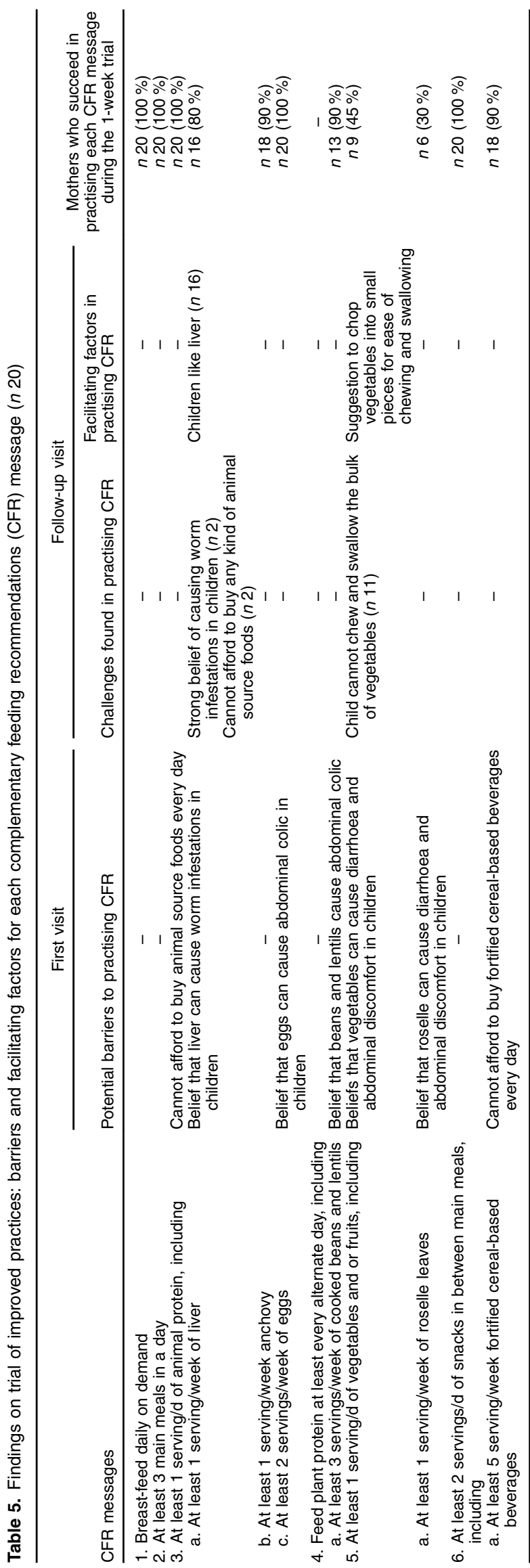

to the CFR. In total, $80 \%$ of the mothers fed their child chicken liver and realised that their child liked liver. These mothers were also willing to continue this practice and to share the CFR messages with other mothers.

\section{Discussion}

This study was the first to comprehensively describe the dietary patterns of children under 2 years of age in Myanmar and to develop CFR using LP analysis. Their current complementary feeding practices cannot provide the adequate level of nutrient requirement for the children. The two modelled best diets (module II analysis, Table 3) suggest that the nutrient content of diets can be improved as long as there is an increase in the number of servings per week of animal source foods, vegetables and legumes compared with observed median intake levels. However, the results showed that optimised CFR based on these food patterns and nutrient-dense locally available foods cannot ensure dietary adequacy especially for the five 'problem nutrients' ( $\mathrm{Ca}, \mathrm{Zn}$, niacin, folate and $\mathrm{Fe}$ ) that are low in the local food supply. Even with the promotion of nutrientdense foods, in these CFR, the worst-case scenario levels of seven nutrients remained $<50 \%$ of their RNI. Dietary adequacy (i.e. achieving $>65 \%$ RNI in the worst-case scenario diet without CFR, module III analysis, Table 3) could only be ensured for protein, vitamin $\mathrm{C}$, riboflavin and vitamin $\mathrm{B}_{12}$. Thus, alternative interventions to complement the CFR are required to ensure dietary adequacy for other nutrients. Nevertheless, the local food-based CFR developed in this study can still improve local dietary practices, although the level of nutrients could not fully reach the recommended daily intake level. TIP suggest that the local food-based CFR are feasible and acceptable for the study population as long as some challenges such as misbeliefs on feeding liver and vegetables are overcome.

The dietary patterns described for the children under 2 years of age in Myanmar are based on 7-d food consumption data. Their typical dietary pattern includes fewer servings per week of animal proteins, vegetables, legumes, nuts and seeds as compared with younger infants from Indonesia and Cambodia ${ }^{(7,16)}$. Using the LP approach, foods rich in the problem nutrients are identified and these foods are incorporated to develop alternative sets of CFR. Totally, twelve alternative sets of CFR were produced and a final set of CFR with the highest nutrient level at the lowest cost was selected. The final CFR achieved the required level for problem nutrients ranging from $26 \%$ (niacin) to $46 \%$ (Ca) at an affordable cost of 271 kyats/d (approximately $0 \cdot 29 \mathrm{USD} / \mathrm{d}$ ) (Table 3). These findings show that LP is a promising problem-solving approach to guide decisions regarding the selection of realistic CFR based on locally available foods to improve the nutrient content in the diet of children.

The number of problem nutrients identified in the complementary feeding diets of Myanmar children is higher than that reported in studies from other countries ${ }^{(7,15,16)}$. Fe, Zn, Ca and niacin have been identified as problem nutrients in previous studies using the LP approach ${ }^{(7,16,27)}$, but folate, which was a problem nutrient in our study, was rarely reported as a problem nutrient except in a subpopulation in Indonesia ${ }^{(28)}$. In our study population, there was a low consumption of folate-rich foods, 
such as red meat, organ meat and vegetables. A recent study conducted in Myanmar also showed that anaemic adolescent school girls had inadequate dietary folate intake, which was consistent with the high prevalence of low serum folate level among these girls ${ }^{(29)}$. Previous research on the adequacy of micronutrient concentrations in industrially manufactured complementary foods showed that most of the processed complementary foods in many countries of Asia are fortified with at least one micronutrient ${ }^{(30)}$. Unlike these countries, lack of fortification programmes for complementary food products in Myanmar could partly explain folate as the limiting nutrient in the complementary foods of Myanmar children.

The prevalence of anaemia among Myanmar children under 5 years of age was $75 \%$ in $2005^{(31)}$. The identified problem nutrients of Fe and folate suggest that poor dietary patterns were likely to have caused deficiency of either Fe or folate or both, which would contribute to nutritional anaemia ${ }^{(32)}$ among preschool children in Myanmar. Anaemia also remains a public health problem among young children in Indonesia, Cambodia and Vietnam ${ }^{(11,33,34)}$, and poor complementary feeding practices are also prevalent in these countries ${ }^{(7,16,35)}$. Anaemia in early childhood has long-term consequences on their growth and development ${ }^{(36,37)}$. It is therefore crucial to improve feeding practices to help address anaemia and other forms of undernutrition in SEA. However, a comprehensive package of interventions will be required, including hygiene and sanitation interventions, to reduce the prevalence of worm infestations and other gastrointestinal infections that can restrict nutrient absorption ${ }^{(38,39)}$.

The findings showed that stunting, underweight and wasting were present as moderate public health problems. Stunting is an indicator of chronic dietary inadequacy of essential type II nutrients ${ }^{(40)}$. The high prevalence of stunting in this study might be due to the poor quality of the complementary feeding diet, in which the growth-limiting nutrients $\mathrm{Zn}, \mathrm{Ca}, \mathrm{P}$ and $\mathrm{Mg}^{(40)}$ are inadequate, although the levels of the latter two nutrients were not analysed by Optifood in the present study. The observed intake of $\mathrm{Zn}$ and $\mathrm{Ca}$ among the children could meet only about $30 \%$ of the RNI (Table 3). Childhood undernutrition has several adverse effects on later life, especially stunting, which when occurs in early life cannot be reversed ${ }^{(41)}$. Therefore, it is important to fill the gap of these problem nutrients to reduce the risk of growth faltering among these children.

The potential food sources identified to partly fill the gap of problem nutrients were chicken liver, anchovy, roselle leaves, fortified cereal-based beverages, cooked beans, lentils and eggs. Chicken liver and anchovies have been identified as nutrient-rich foods for improving the dietary adequacy of Indonesian infants ${ }^{(7,42)}$; however, roselle has never been identified in other countries as a potential nutrient-dense food. Although these nutrient-dense foods are easily available in the study area, the amount and frequency of consumption is very low, especially of liver and roselle leaves (Tables 1 and 2). Our study suggests that negative food beliefs and taboos about these foods account for their low level of intake. Misbeliefs regarding liver as a cause of worm infestation and about vegetables and legume as causes of diarrhoea need to be addressed when promoting CFR. Our results, however, suggested that some of these misbeliefs can be overcome (i.e. $80 \%$ of mothers fed liver to their child during TIP and they realised that their children liked liver), although such foods are rarely fed to the study population. In contrast, our findings suggest that intensive education efforts will be needed to increase vegetable intakes, perhaps educating the community about food preparation methods that improve the taste of vegetables for children while preventing nutrient loss during cooking.

Myanmar food-based dietary guidelines (FBDG) included only three generic messages for infant and young child feeding: (1) exclusive breast-feeding for 6 months; (2) introduction of complementary food starting from 6 months of age; and (3) feeding the child a variety of food groups. There is no specific information on the type, amount and frequency of foods to be fed to the child. The LP approach helps identify the specific food items, food groups or food subgroups that could be promoted to best fill nutrient gaps in the diets of these children to complement the current FBDG. Moreover, LP is very useful for countries such as Myanmar where population-specific local food-based recommendations (FBR) could significantly improve the dietary pattern and nutrient intakes among vulnerable age groups. Local-specific FBR might also be required, particularly in countries with diverse food patterns across populations. In Myanmar, although rice is the main staple food throughout the country, dietary patterns are different to a certain extent across populations on the basis of ethnicity, as well as due to differences in locally and/or seasonally available foods.

Several studies have used the LP approach to develop $\operatorname{FBR}^{(7,16,25)}$, but to our knowledge the acceptability of FBR developed by the LP approach has not yet been reported using the TIP. TIP have been used in other programmes to improve health-promoting behaviours such as family planning, prevention of malaria, and control of indoor air pollution. TIPs help us identify the socio-economic barriers and cultural food beliefs/ taboos that must be overcome to promote positive deviant behaviour that will improve complementary feeding practices. TIPs also educate mothers to the fact that they can feed their children with liver and vegetables without any harm as long as their hygiene and sanitation practices are good and reduce the unnecessary cost of less-nutritious snacks and drinks. By setting upper level model constraints at the 95th or 99th percentile of observed intakes, we have based the selection of CFR on a positive deviant approach in which the most promising nutrientdense foods, which may be consumed by very few people, are selected to be promoted in a set of CFR for the population.

The user-friendly software Optifood used in our study simplifies the complex process of running the LP models used in this study and reduces the chance of error. Our goal is to develop local-specific CFR/FBR in each district (state/region level) and Optifood analysis can be run at the district level by the staff from state/region nutrition teams after training. Another strength of our study was the development of a food composition table for the local foods in the study areas. The nutrient content of local recipes was averaged across ten mothers to minimise the selection bias when calculating the nutrient composition of local recipes.

Despite these strengths, our study also has some limitations. First, the study was conducted in a delta region with high prevalence of childhood undernutrition, which limits generalisation of the findings on problem nutrients and hence 
acceptable nutrient-dense foods in other regions of Myanmar. In addition, we used the 95th or 99th percentile for some foods, as we believed these foods could be successfully promoted. However, their ease of adoption may be more difficult than if a more conservative selection criterion such as foods consumed by $>10 \%$ of the target population (i.e. a 90th percentile limit) had been used. Finally, the nutrient composition of the food items modelled in this study was taken from different food composition databases. The nutrient composition of foods is known to vary across regions and countries ${ }^{(17,43)}$, which might have affected our results, especially if the nutrient composition data of foods promoted, in the final CFR, were not accurate.

In conclusion, the dietary practices of 12-23-month-old children living in the Ayeyarwady Region of Myanmar are poor. Their diet can be improved using locally available foods by using LP analysis. However, it will be difficult to ensure dietary adequacy for the five 'problem nutrients' ( $\mathrm{Ca}, \mathrm{Zn}$, niacin, folate and Fe) using only locally available food. The final CFR based on realistic amounts of nutrient-dense locally available foods (i.e. liver, roselle leaves, anchovies, eggs, beans and fortified cereal-based beverages) would only ensure dietary adequacy for five nutrients (i.e. protein, riboflavin, and vitamins $\mathrm{C}, \mathrm{A}$ and $\mathrm{B}_{12}$ ). The results of $\mathrm{LP}$ analysis suggest alternative strategies such as the following: fortification of complementary food products with $\mathrm{Ca}, \mathrm{Zn}$, niacin, folate and Fe to fill the gap to meet the recommended level of intake; multi-micronutrient sprinkles for 6-35-month-old children; or fortification of staple foods with multiple micronutrients such as rice. TIP showed it was possible to overcome the socio-cultural barriers against feeding young children liver but those against feeding vegetables will be more difficult to address. These findings highlighted the usefulness, for nutrition programme planners, of combining the LP and TIP approaches to inform decisions before implementing a foodbased intervention. The diet patterns should be assessed in other seasons as well as in other areas where undernutrition is less problematic in Myanmar in order to identify problem nutrients and develop population-specific food-based CFR. The long-term sustainability of the CFR developed in this study should be tested, and the actual change in nutrient intake levels after successful adoption of these CFR should be further investigated in future intervention studies.

\section{Acknowledgements}

The authors thank the Department of Health, Ministry of Health, Myanmar, for giving the permission to conduct this study. The authors also thank the local government officials for their cooperation during the field work. The authors acknowledge all the mothers and children who participated in the study. The authors greatly appreciate Htin Linn from the National Nutrition Center for his technical inputs during the field work and May Khin Than from the National Nutrition Center for her support in the study. The authors acknowledge Kyaw Swar Mya from University of Medicine 1 for his support during dietary data collection and Ohnmar Kyi from the Department of Health Planning for her support in obtaining health profile data. The authors thank Khin Moe Moe Aung and Swe Mar Hlaing for their assistance during data collection.
This work was supported by the DAAD (German Academic Exchange Service) scholarship and the Nestlé Foundation.

L. M. H., U. F., B. U. and A. F. conceptualised the study; L. M. H. and M. K. H. compiled the sources for the food composition database; L. M. H. prepared the protocol and performed the data collection; U. F. supervised the data collection; L. M. H., U. F., E. L. F. and M. K. H. ran Optifood and interpreted the data; L. M. H. wrote the draft manuscript; and U. F., M. K. H., B. U., A. F. and E. L. F. contributed to editing of the manuscript.

The authors declare that there are no conflicts of interest.

\section{References}

1. Dewey KG \& Vitta BS (2013) Strategies for Ensuring Adequate Nutrient Intake for Infants and Young Children During the Period of Complementary Feeding. Washington: Alive \& Thrive.

2. Fahmida U \& Preedy VR (2012) Food-Based Complementary Feeding and Its Impact on Growth: Southeast Asian Perspectives: Handbook of Growth and Growth Monitoring in Health and Disease. New York: Springer.

3. Gibson RS, Bailey KB, Gibbs M, et al. (2010) A review of phytate, iron, zinc, and calcium concentrations in plant-based complementary foods used in low-income countries and implications for bioavailability. Food \& Nutrition Bulletin 31, Suppl. 2, 134-146.

4. Kikafunda J, Walker A \& Tumwine J (2003) Weaning foods and practices in central Uganda: a cross-sectional study. Afr J Food Agric Nutr Dev 3, 0-0.

5. Castle S, Yoder PS, Konaté MK, et al. (2001) Introducing Complementary Foods to Infants in Central Mali. MEASURE DHS+, ORC Macro. Calverton, MD: ORC Macro.

6. Hotz C \& Gibson R (2004) Participatory nutrition education and adoption of new feeding practices are associated with improved adequacy of complementary diets among rural Malawian children: a pilot study. Eur J Clin Nutr 59, 226-237.

7. Santika O, Fahmida U \& Ferguson EL (2009) Development of food-based complementary feeding recommendations for 9- to 11-month-old peri-urban Indonesian infants using linear programming. J Nutr 139, 135-141.

8. Anderson VP, Cornwall J, Jack S, et al. (2008) Intakes from non-breastmilk foods for stunted toddlers living in poor urban villages of Phnom Penh, Cambodia are inadequate. Matern Child Nutr 4, 146-159.

9. Gibson RS, Ferguson EL \& Lehrfeld J (1998) Complementary foods for infant feeding in developing countries: their nutrient adequacy and improvement. Eur J Clin Nutr 52, 764-770.

10. Winichagoon P (2008) Coexistence of micronutrient malnutrition: implication for nutrition policy and programs in Asia. Asia Pac J Clin Nutr 17, Suppl. 1, 346-348.

11. Anderson VP, Jack S, Monchy D, et al. (2008) Co-existing micronutrient deficiencies among stunted Cambodian infants and toddlers. Asia Pac J Clin Nutr 17, 72-79.

12. Dewey KG \& Brown KH (2003) Update on Technical Issues Concerning Complementary Feeding of Young Children in Developing Countries and Implications for Intervention Programs. Geneva: WHO.

13. World Health Organization \& United Nations International Children's Emergency Fund (2003) Global Strategy for Infant and Young Child Feeding. Geneva: WHO.

14. Ferguson EL, Darmon N, Briend A, et al. (2004) Food-based dietary guidelines can be developed and tested using linear programming analysis. J Nutr 134, 951-957. 
15. Ferguson EL, Briend A \& Darmon N (2008) Can optimal combinations of local foods achieve the nutrient density of the F100 catch-up diet for severe malnutrition? J Pediatr Gastroenterol Nutr 46, 447-452.

16. Skau JK, Bunthang T, Chamnan C, et al. (2014) The use of linear programming to determine whether a formulated complementary food product can ensure adequate nutrients for 6- to 11-monthold Cambodian infants. Am J Clin Nutr 99, 130-138.

17. Dewey KG, Cohen RJ \& Rollins NC (2004) Feeding of nonbreastfed children 6-24 months of age in developing countries. Food Nutr Bull 25, 377-402.

18. Ministry of National Planning and Economic Development \& Ministry of Health (2010) Monitoring the Situation of Children and Women, Multiple Indicator Cluster Survey (MICS) 20092010, Nation Wide Survey, Report. Nay Pyi Taw: Myanmar MoH.

19. World Health Organization \& Food and Agricultural Organization (2004) Vitamin and Mineral Requirements in Human Nutrition, 2nd ed. Geneva: FAO and WHO.

20. Gibson RS (2005) Principles of Nutritional Assessment. New York: Oxford University Press.

21. Fahmida U, Rumawas JSP, Utomo B, et al. (2007) Zinc-iron, but not zinc-alone supplementation, increased linear growth of stunted infants with low haemoglobin. Asia Pac J Clin Nutr 16, 301-309.

22. World Health Organization (2006) World Health Organization Multicentre Growth Reference Study Group. WHO Child Growth Standards: Length/Height-for-Age, Weight-Forage, Weight-for-Length, Weight-for-Height and Body Mass Indexfor-Age: Methods and Development. Geneva: WHO.

23. World Health Organization (2003) Guiding Principles for Complementary Feeding of the Breastfed Child. Geneva: Pan American Health Organization WHO.

24. Bunch S \& Murphy S (1997) User's Guide to the Operation of the WorldFood Dietary Assessment System, Version 2.0. Berkeley, CA: Office of Technology Licensing.

25. Daelmans B, Ferguson E, Lutter CK, et al. (2013) Designing appropriate complementary feeding recommendations: tools for programmatic action. Matern Child Nutr 9, Suppl. 2, 116-130.

26. Brown KH, Rivera J, Bhutta Z, et al. (2004) International Zinc Nutrition Consultative Group (IZiNCG) technical document ${ }^{\#} 1$. Assessment of the risk of zinc deficiency in populations and options for its control. Food Nutr Bull 25, Suppl. 2, S99-S203.

27. Food and Nutrition Technical Assistance (2013) Development of Evidence-Based Dietary Recommendations for Children, Pregnant Women, and Lactating Women Living in the Western Highlands of Guatemala: Summary Report. Washington, DC: FHI 360/FANTA.

28. Fahmida U, Santika O, Kolopaking R, et al. (2014) Complementary feeding recommendations based on locally available foods in Indonesia. Food Nutr Bull 35, Suppl. 3, 174S-179S.
29. Htet MK, Fahmida U, Thurnham DI, et al. (2015) Folate and vitamin $B_{12}$ status and dietary intake of anaemic adolescent schoolgirls in the delta region of Myanmar. $\mathrm{Br} J \mathrm{Nutr}$ (epublication ahead of print version 20 October 2015).

30. Gibbs M, Bailey KB, Lander RD, et al. (2011) The adequacy of micronutrient concentrations in manufactured complementary foods from low-income countries. J Food Compost Anal 24, 418-426.

31. Ministry of Health (2007) Health in Myanmar 2007. Nay Pyi Taw: MOH.

32. Badham J, Zimmermann MB \& Kraemer K (2007) In The Guide Book for Nutritional Anemia, 52 pp. [J Badham, MB Zimmermann and K Kraemer, editors]. Basel, Switzerland: Sight and Life Press.

33. Sandjaja S, Budiman B, Harahap H, et al. (2013) Food consumption and nutritional and biochemical status of 0 5-12-year-old Indonesian children: the SEANUTS study. Br J Nutr 110, Suppl. 3, S11-S20.

34. Le Nguyen BK, Le Thi H, Nguyen Do VA, et al. (2013) Double burden of undernutrition and overnutrition in Vietnam in 2011: results of the SEANUTS study in 0 5-11-year-old children. Br J Nutr 110, Suppl. 3, S45-S56.

35. Dibley MJ, Senarath U \& Agho KE (2010) Infant and young child feeding indicators across nine East and Southeast Asian countries: an analysis of National Survey Data 2000-2005. Public Health Nutr 13, 1296-1303.

36. Hurtado EK, Claussen AH \& Scott KG (1999) Early childhood anemia and mild or moderate mental retardation. Am J Clin Nutr 69, 115-119.

37. Ramakrishnan U (2001) Functional consequences of nutritional anemia during pregnancy and early childhood. In Nutritional Anemias, pp. 43-68 [I Wolinsky and JF Hickson Jr, editors]. New York and Washington, DC: CRC Press.

38. Ngui R, Lim YAL, Kin LC, et al. (2012) Association between anaemia, iron deficiency anaemia, neglected parasitic infections and socioeconomic factors in rural children of West Malaysia. PLOS Negl Trop Dis 6, e1550.

39. Annibale B, Capurso G \& Delle Fave G (2002) Consequences of Helicobacter pylori infection on the absorption of micronutrients. Dig Liver Dis 34, Suppl. 2, S72-S77.

40. Golden MHN (2009) Specific deficiencies versus growth failure: type I and type II nutrients. J Nutr Environ Med 6, 301-308.

41. Dewey KG \& Begum K (2011) Long-term consequences of stunting in early life. Matern Child Nutr 7, 5-18.

42. Ferguson EL, Darmon N, Fahmida U, et al. (2006) Design of optimal food-based complementary feeding recommendations and identification of key 'Problem Nutrients' using goal programming. J Nutr 136, 2399-2404.

43. Merchant A \& Dehghan M (2006) Food composition database development for between country comparisons. Nutr J 5, 2. 